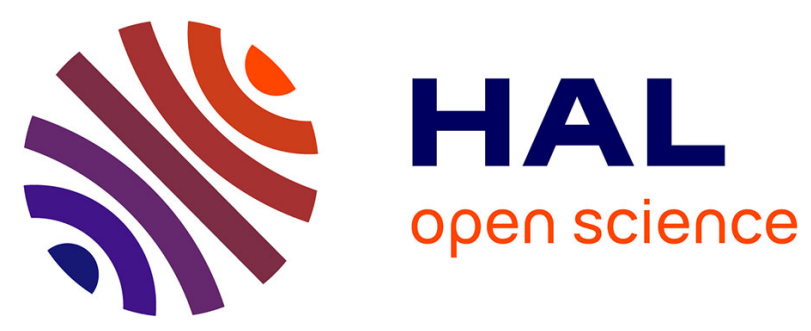

\title{
Coalescence of lenticular anticyclones in a linearly stratified rotating fluid
}

\author{
A Orozco Estrada, Raúl C Cruz Gómez, A Cros, P Le Gal
}

\section{To cite this version:}

A Orozco Estrada, Raúl C Cruz Gómez, A Cros, P Le Gal. Coalescence of lenticular anticyclones in a linearly stratified rotating fluid. Geophysical and Astrophysical Fluid Dynamics, 2020, 114 (4-5), 10.1080/03091929.2020.1734199 . hal-03190915

\section{HAL Id: hal-03190915 https://hal-amu.archives-ouvertes.fr/hal-03190915}

Submitted on 6 Apr 2021

HAL is a multi-disciplinary open access archive for the deposit and dissemination of scientific research documents, whether they are published or not. The documents may come from teaching and research institutions in France or abroad, or from public or private research centers.
L'archive ouverte pluridisciplinaire HAL, est destinée au dépôt et à la diffusion de documents scientifiques de niveau recherche, publiés ou non, émanant des établissements d'enseignement et de recherche français ou étrangers, des laboratoires publics ou privés.

\section{(ㅇ)(1) $\$$}

Distributed under a Creative Commons Attribution - NonCommercial - NoDerivatives $\mid 4.0$ 
Geophysical and Astrophysical Fluid Dynamics

Vol. 00, No. 00, 00 Month 2019, 1-17

\title{
Coalescence of lenticular anticyclones in a linearly stratified rotating fluid
}

\author{
A. Orozco Estrada†, Raúl C. Cruz Gómez†, A. Crosł and P. Le Gal‡* \\ †Physics Department, CUCEI, Universidad de Guadalajara, Blvd. Gral. Marcelino García Barragán \\ 1421, Olímpica, 44430 Guadalajara Jal., Mexico \\ ‡, Aix Marseille Université, CNRS, Centrale Marseille, IRPHE, 49 Rue F. Joliot Curie, \\ Technopôle de Chateau-Gombert, Marseille, France \\ (Received 00 Month 20xx; final version received 00 Month 20xx)
}

\begin{abstract}
This study is devoted to laboratory experiments on the coalescence of two lenticular anticyclones in a linearly stratified rotating fluid. These anticyclones are generated by injecting small volumes of fluid at the center of a rotating tank where a linearly stratified layer was previously prepared with salt. The characteristics of the interaction between the vortices are studied by visualization and Particle Image Velocimetry (PIV) as a function of the initial separation distance between the vortices, the Coriolis parameter of the rotating table and the Brünt-Väisälä frequency of the density stratification. Our results show that the merging critical distance depends drastically on the Rossby radius of deformation of the vortices and are in complete agreement with previous numerical modeling of vortex coalescence. We have also observed that mergers involve three-dimensional processes as the vortices intertwine together possibly because of the presence of an elliptic instability that tilts the vortex cores. They are also accompanied by the emission of vorticity filaments and internal gravity waves radiation although we cannot prove that in our experiments these waves are solely due to the merging process.
\end{abstract}

\section{Introduction}

Oceanic meso-scale lenticular vortices play an important role in the redistribution of momentum, heat, salt, nutrients and plankton in oceans and thus contribute to the climate equilibrium and ecological diversity on Earth. These vortices are governed by geostrophic and hydrostatic balances between pressure gradients, Coriolis and buoyancy forces from where they get their shape and aspect ratio as shown by Hassanzadeh et al. (2012), Aubert et al. (2012) and by De la Rosa Zambrano et al. (2017) for surface vortices. Their chaotic motions in oceans give rise to a quasi-geostrophic turbulence characterized by a reverse energy cascade and a forward enstrophy cascade (Kraichnan 1967, Charney 1971). Understanding the way energy escapes from this mesoscopic turbulence to feed the smallest oceanic scales where dissipation occurs, is the subject of an intense research. In this context, vortex coalescence as observed in oceans (Schultz Tokos et al. 1994) can be seen as a building block of these reverse and forward cascades: vortex mergers participate to the reverse cascade towards the large scales of the oceanic turbulence while they generate at the same time fine filaments of vorticity (Cushman-Roisin 1989) as well as internal waves (Viudez 2006, Reinaud and Dritschel 2018) that could feed small dissipative scales. The aim of the present study is to describe and parametrize the merging of two lenticular anticyclones by means of stratified flow experiments performed on a rotating table. For this purpose, we generate pairs of anticyclonic vortices by the gentle injection of two small volumes of water inside a continuously stably stratified rotating layer in a similar way that Griffiths and Hopfinger (1987) did thirty years ago but for a two layer system. To our knowledge, this problem has never been revisited experimentally for the case of lenticular vortices imbedded in

*Corresponding author. Email: legal@irphe.univ-mrs.fr 
a continuously stratified layer.

Symmetric vortex pairing has been widely studied in two-dimensional, inviscid, incompressible flows (Zabusky et al. 1979, Melander et al. 1988, Waugh 1992, Nielsen et al. 1996). It was shown that, in this case, the vortices merge when the ratio of the initial distance $d_{0}$ between their centers and their radius $R$ is small enough (typically $d_{0} / R \leq 3.3$, Waugh (1992), Meunier et al. (2005), Leweke et al. (2016)). Nevertheless, when the vortices are baroclinic, the Rossby radius of deformation may drastically modify the critical distance $d_{c}$ for which merging occurs. The Rossby radius of deformation $\lambda$ gives an order of magnitude of the scale for which the Coriolis effect becomes as important as the buoyancy. It gives the effective length of the horizontal distance between the region of generation of a disturbance and its outermost extent. This Rossby radius of deformation is equal to:

$$
\lambda=\frac{N}{f} H,
$$

where $H$ is the vortex height, $f$ the Coriolis parameter and $N$ the Brünt-Väisälä frequency. For a continuously stratified fluid, $N=\sqrt{-\frac{g}{\rho_{0}} \frac{\partial \rho}{\partial z}}$ represents the oscillation frequency of a parcel of fluid with density $\rho_{0}$ vertically displaced in a fluid layer of density $\rho(z)$ and where $g$ is the gravity.

As far as we know, the first and unique experimental study on baroclinic vortex pairing in a two-layer stratification was carried out by Griffiths and Hopfinger (1987) for various values of $\lambda / R$ and $d_{0} / R$. The vortex height is fixed by the top layer depth and it was found that a strong enough stratification $\lambda / R>2.0$ enhances the merging, and in consequence increases the critical distance $d_{c}$ that separates pairing to non pairing cases. Following this experimental work, two numerical studies evidenced the importance of the initial conditions on the influence of stratification in a two-layer model. First, Polvani et al. (1989) found that stratification does not modify the critical initial distance $d_{c}$; but this contradictory result was explained a year later by Verron et al. (1990) who proved that density stratification has indeed a drastic effect on $d_{c} / R$ if the relative vorticity is zero in the lower layer. This condition was not fulfilled in the work of Polvani et al. (1989) who enforced a zero potential vorticity in the lower layer. Verron et al. (1990) found that the barotropic limit is reached when $\lambda / R \rightarrow 0$ as well as for large values of $\lambda / R(\lambda / R>4)$. In the case of zero relative vorticity initialization, the merging is more efficient when $\lambda / R \simeq 2.1$ as the critical distance reaches a maximum value $d_{c} \simeq 6.8 R$. Nevertheless, it was noted later by Verron and Valcke (1994) that this maximum can be modified if mixed conditions between zero potential and zero relative vorticity initialization are taken into account. Indeed the peak in the initial distance critical values can shift towards lower values of $\lambda / R$ and $d_{c} / R$.

Merger observations in the Atlantic Ocean of strongly interacting meddies were reported by Carton et al. (2010) and L'Hégaret et al. (2014). Some mergers were also described by Schultz Tokos et al. (1994), Carton et al. (2002), Rodríguez-Marroyo et al. (2011) and Barbosa et al. (2013). Besides these oceanographic observations, merging of two giant anticyclones in the atmosphere of Jupiter is also described by Sanchez-Lavega et al. (2001). We can moreover easily imagine other astrophysical applications such as the coalescence of baroclinic vortices in accretion disks for instance. Whereas the two-layer model is a good first order approximation for these natural cases, oceans or atmospheres are continuously stratified. To our knowledge, only one laboratory study has been reported up to now in a linearly stratified rotating fluid but only for pairs of barotropic vortices (Cariteau 2005). This laboratory experiment showed that the critical distance for the merging of long columnar vortices increases when stratification increases in agreement with the numerical prediction of Dritschel (2002), who extended the results of Von Hardenberg et al. (2000) to vortices with large aspect ratio $H / R=20 \mathrm{f} / N$. In these last studies, three-dimensional quasi-geostrophic vortices are modeled and the barotropic 
critical distance is recovered for flat vortices $(H / R \rightarrow 0)$, whereas unexpectedly $d_{c} / R$ continues to increase with $H / R$. This enhancement of coalescence for large separating distances is due to the existence of a bending instability (most probably the zig-zag instability of Billant (2010), Otheguy et al. (2015)) that brings the vortex cores closer together. Another ingredient that was pointed by Valcke and Verron (1997) comes from the possible presence of shielding rings around the vortices. Indeed, as observed numerically in the pairing of realistic oceanic meddies by Ciani et al. (2016), the cyclonic ring around each anticyclone can prevent the merger by a redistribution of the shield cyclonic potential vorticity that generates a repulsive effect on the cores. As we will see later, our laboratory vortices are indeed shielded and our experiments reveal some interesting features already detected in numerical simulations although the shields seem not to prevent coalescence in our present observations.

Our study explores the $\left(\lambda / R\right.$ and $\left.d_{0} / R\right)$ space in order to determine the critical merging distance $d_{c}$ as a function of the Rossby radius of deformation $\lambda$. Both the global rotation rate and the density stratification will be changed. Around ninety experimental runs (including Particle Image Velocimetry (PIV) measurements and dye visualizations) have been realized. Each experiment is labeled with a number which will be used to identify them. Pairing and non pairing situations are discriminated by measuring the evolution in time of the distance separating the vortex cores. When this separation distance becomes less than a vortex diameter, the merging process will be declared effective. In most of the runs, there are no ambiguities to decide if the pairs have merged or not after a duration of about 20 minutes per run. Besides determining the critical initial distance $d_{c}$, we have also observed that the injection flux that controls the Rossby number of the vortices, plays a role on the merging time and on the rotation rate of the pair. These results are consistent with the recent numerical simulations of Reinaud and Dritschel (2018). Finally, we will show that a Fourier analysis permits the detection of Internal Gravity Waves (IGWs) that radiate energy away during the vortex generation by injection and during the first instants of coalescence.

\section{Experimental set-up and measurement techniques}

\section{1. $\quad$ Rotating table installation and methodology}

All the experiments were performed in the Geophysical Fluid Dynamics Laboratory of Guadalajara University. The experimental set-up is shown in figure 1. The rotating transparent Plexiglass tank dimensions are $40 \times 40 \times 40 \mathrm{~cm}^{3}$ which are at least ten times larger than the vortex diameters and twenty times deeper that the vortex heights. Two constant density $5 \mathrm{~cm}$ deep layers are generated at the bottom and at the top of the total fluid layer, while the stratified region has a vertical extension of $20 \mathrm{~cm}$. The upper layer water is fresh while the bottom layer water is salty and has a density whose value depends upon the desired stratification. The linear stratification is made by the two-bucket method (Oster 1965) which is a classical method that consists in mixing progressively the water from a bucket of pure water with the brine of a second bucket. Then the Brünt-Väisälä frequency $N$ is determined from the measurement of the density gradient after the fluid was left at rest for more than 12 hours before performing the experiments. The diffusion of salt in water being slow (Schmidt number equal to 700), the stratified layer can be kept for days if not perturbed. The whole device shown in figure 1 is then slowly set into rotation for more than 2 hours until the fluid layer reaches a solid body rotation at a rotation rate $\Omega$. The Coriolis parameter is given by $f=2 \Omega$. A side-view camera allows to determine the depth $H$ of the vortices as shown in figure 1, while the top-view camera records the two vortices evolution after they have been created.

The two anticyclones are generated by injecting a small volume of fluid, between $5 \mathrm{ml}$ and $20 \mathrm{ml}$, at the center of the vertical stratified layer (see for instance Hopfinger and van Heijst (1993)) as it is sketched in figure 1. In order to generate controlled vortices, two micro-pumps 
suck dyed salty water from two distinct tanks whose density is equal to the density in the middle-plane of the stratified layer. Two flow meters allow to measure the flow rate. The dyed water is injected through two rigid $4 \mathrm{~mm}$ inner diameter pipes which are fixed on the rotating tank. The separation distance $d_{0}$ between the pipes sets the initial distance between the two vortices. Note that some of our experiments were realized by hand injection, using syringes (instead of the pumps), of a $5 \mathrm{ml}$ volume of water in approximately $12 \mathrm{~s}$. As already explained in the introduction, each run is labelled by a number and is characterized by its rotation Coriolis parameter $f$, its Brünt-Väisälä frequency $N$, the initial separating distance $d_{0}$ and also the flow rate $Q$ of the injection that has, as we will show later, a great influence on the Rossby number of the vortices.

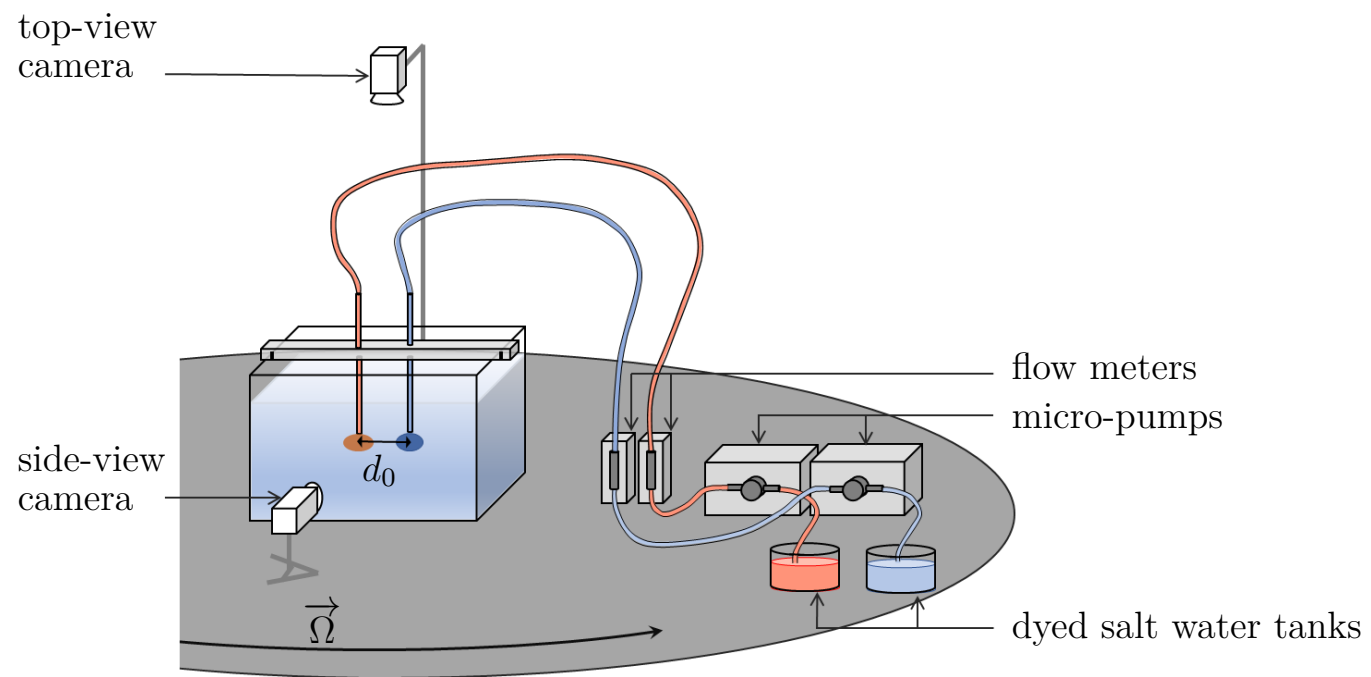

Figure 1. Experimental system scheme with the pipes, tubes and injection pumps that permit the creation of the pair of anticyclones.

Most of the time, both vortices in the pairs were of equal strength and size. However, in about one third of the total number of experiments, the symmetry between both vortices is not perfectly respected (because of uncontrolled experimental uncertainties like tiny bubbles in the injection pipes for instance) and asymmetric fusion takes place (Dritschel 2002). In this case, one of the vortices wraps around the other one as seen in figure 2. Therefore in the following we have removed all the data corresponding to these asymmetric experimental configurations and we are able to produce a state diagram using more than forty data points.
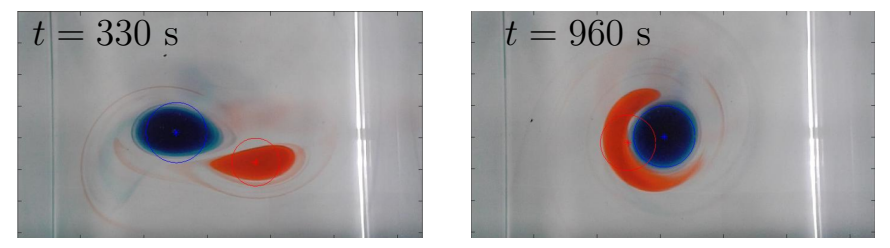

Figure 2. Example of an asymmetric merging for $f=1 \mathrm{rad} / \mathrm{s}, N=1.71 \mathrm{rad} / \mathrm{s}, Q=60 \mathrm{ml} / \mathrm{min}$ and $d_{0}=14 \mathrm{~cm}$.

\subsection{Visualization and PIV measurements}

Most of our experiments have been realized with vortices colored with dye in order to measure their core diameters and heights. One vortex is colored in red and the other one in green (or blue) in order to facilitate the use of simple algorithms to differentiate and track the vortices 
by video image analysis. Their radius and separating distance are recorded by the top-view camera. Their height is measured by the side-view camera. Radii and heights will be then used to compute the Rossby radius of deformation $\lambda$ of the anticyclones. In order to calculate the Rossby number of the vortices, but also to describe precisely their shape, Particle Image Velocimetry (PIV) measurements were performed in both horizontal and vertical planes for some experiments. To do so, small polyamide particles (diameter of $50 \mu \mathrm{m}$ ) were added in the water prior to the stratification process. These particles are also added in the salt water that is injected to generate the vortices. A green light laser beam is then used to illuminate the particles in planes crossing the vortices. The top view camera is used to record the movement of the particles with a frame rate of $1 \mathrm{~Hz}$. Two frames of the video sequences are used to estimate the particle displacement using PIVlab (Thielicke and Stamhuis 2014) or Flow Manager (Dantec Dynamics $\left.{ }^{(}\right)$softwares. Each image is subdivided in 32 pixels interrogation windows to calculate the instantaneous velocity fields as a function of time at the rate of 1 field per second.

\section{Description of a single anticyclone}

\subsection{The geostrophic balance of the anticyclonic lenses}

In order to describe a single vortex before proceeding to the merging experiments, we have studied its velocity field by performing PIV measurements in its equatorial plane. Figure 3a) presents an example of the vorticity field recorded in and around an anticyclone. As can be clearly seen, a cyclonic ring is present around the vortex core. The Rossby number of the vortex, i.e. its magnitude, can be measured by the minimum of the vorticity field $\omega_{0}$ in its center: $R o=\omega_{0} / f$. This kind of shielded vorticity field is quite classic for oceanic vortices and can be modeled by a modified Gaussian shape:

$$
\omega(r)=\omega_{0}\left[1-\frac{\alpha}{2}\left(\frac{r}{L}\right)^{\alpha}\right] \exp \left[-\left(\frac{r}{L}\right)^{\alpha}\right]
$$

where where $\alpha$ and $L$ are free parameters. When $\alpha=2$, the vortex is said to be Gaussian. Figure 3-b) shows the data from the previous field, plotted along the radius for different azimuthal angle. A quite nice collapse of the vorticity radial profiles proves that they can be fitted with a modified Gaussian function where $\alpha$ and $L$ are determined by best fit:
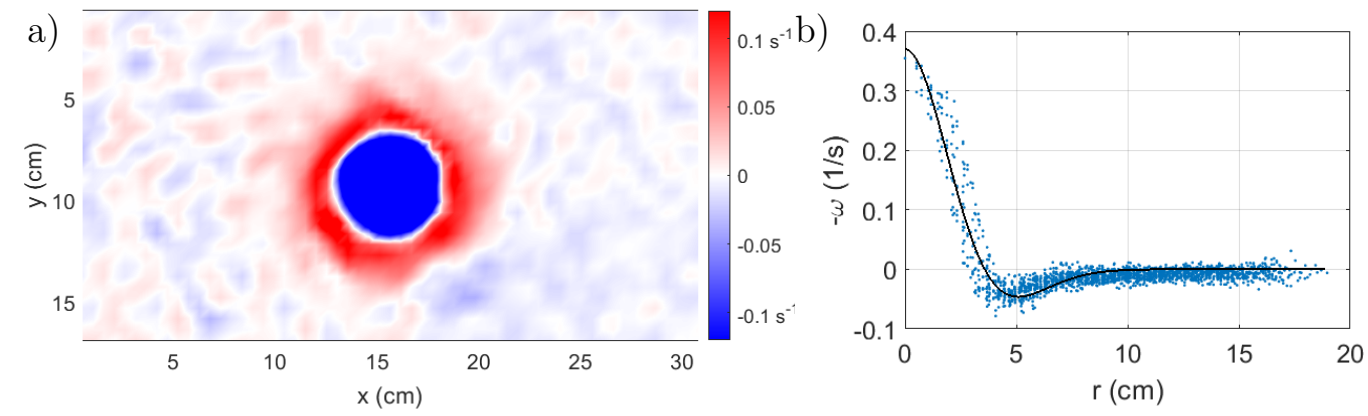

Figure 3. The vorticity field of a single shielded anticyclone in the laboratory for $f=1 \mathrm{rad} / \mathrm{s}, N=1.02 \mathrm{rad} / \mathrm{s}$ and $Q=80 \mathrm{ml} / \mathrm{min}$. b) Vorticity profile showing 1888 data points together with their modified Gaussian fit with $\alpha=1.9$ and $L=3.5 \mathrm{~cm}$ as described in equation (2). 
In this experimental run, $\alpha$ lies between 2 and 1.5 as time varies and this will always be the case whatever the parameter values. $L$ is the vortex typical horizontal scale corresponding to the radial location where the vorticity goes through zero. This value also varies in time as the vortex expands when slowing down. Figure 4 presents the temporal variation of the Rossby number Ro, of $L$ and of $\alpha$ respectively. This slow damping of the vortex has been analysed in great details by Facchini and Le Bars (2016) and is due to a subtle interplay between viscous dissipation and secondary circulations which advect the density anomaly.
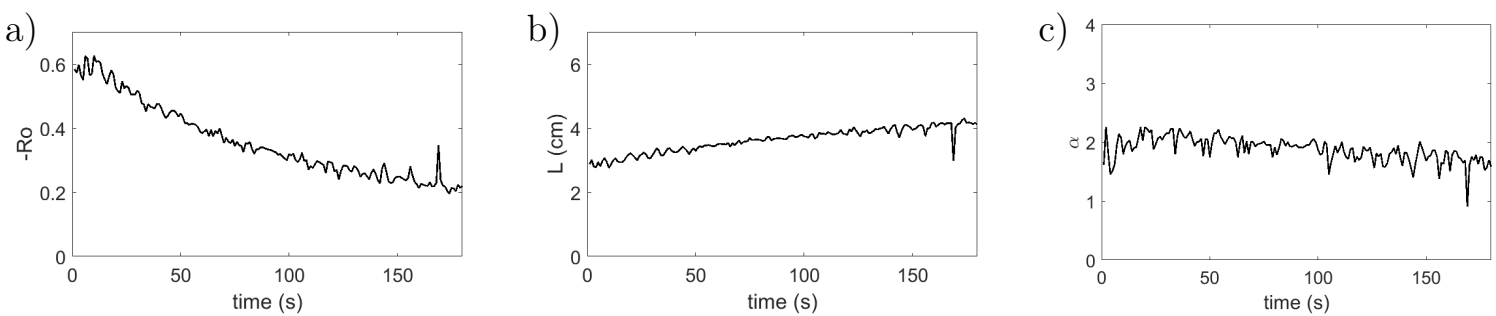

Figure 4. Temporal evolution of the Rossby number Ro (a), of the horizontal typical scale $L$ (b) and of $\alpha$ (c) for the experimental run of figure 3 .

As it was shown in previous studies, vortices are governed by geostrophic and hydrostatic balances from where they get their shape that obeys scaling laws (see De la Rosa Zambrano et al. (2017) for instance). Moreover, we have also observed that the Rossby number depends upon the injection rate as the vorticity is a direct consequence of the horizontal radial flow, which by action of the Coriolis effect generates an azimuthal velocity field. Figure 5 shows this dependence for four values of $Q$, where a linear behaviour is observed.

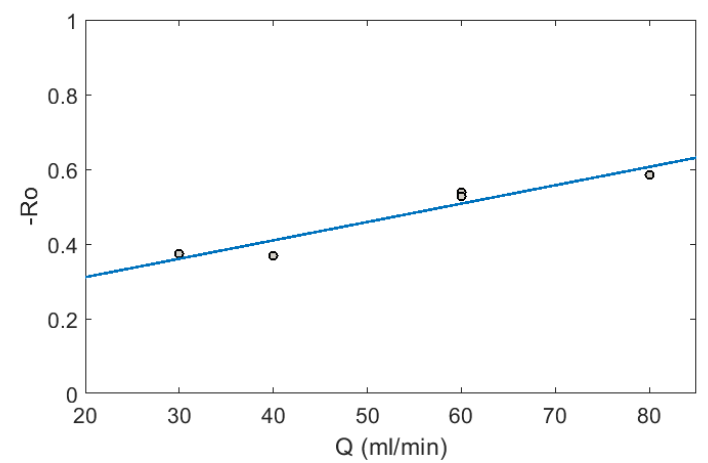

Figure 5. Variation of the Rossby number Ro for a single vortex as a function of the injection flow rate $Q$. Data points for five experiments are plotted. Circles show the mean Rossby number for each experiment.

Finally, to complete our description of a single vortex, we have also realized PIV measurements in vertical planes going through the vortex at mid-radius. As this plane is off centered, because of its thickness $(1 \mathrm{~mm})$, the projection onto the plane of the particle trajectories can be recorded and analyzed. As presented in figure 6 , the evolution of the azimuthal velocity along the vertical direction can be quite nicely fitted by a modified Gaussian function with a typical vertical scale $Z$ and an exponent $\beta$. Therefore our laboratory vortices created by injection of fluid in a rotating and stratified layer have a similar three-dimensional shielded structure as the real oceanic vortices (Ciani et al. 2016): 


$$
\omega(r)=\omega_{0}\left[1-\frac{\alpha}{2}\left(\frac{r}{L}\right)^{\alpha}\right] \exp \left[-\left(\frac{r}{L}\right)^{\alpha}\right] \exp \left[-\left(\frac{z}{Z}\right)^{\beta}\right]
$$
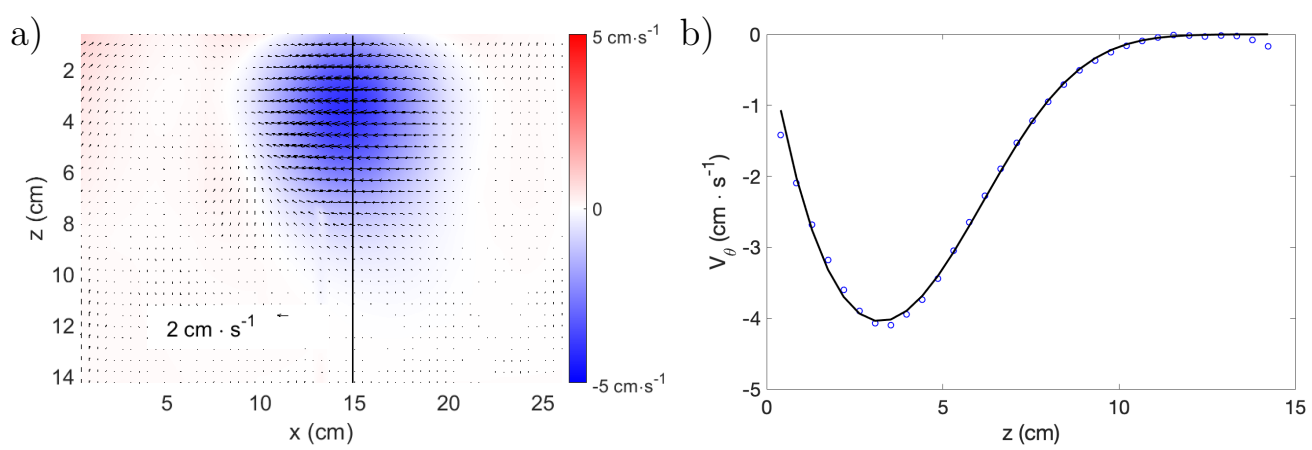

Figure 6. a) The velocity field in a vertical section going through the anticyclone at mid-radius. b) Velocity profile showing the data points together with their modified Gaussian fit with $\beta=1.87$ as described in equation (3).

\subsection{Measurements of the radius and height of the vortices}

Most of our experiments were realized using visualizations of the pair of vortices. After injection, we waited several table rotation periods to get a quasi-geostrophic adjustment of the injected patches during which these get their circular shape. Their radii $R$ were then obtained at this initial time by a measurement of the horizontal size of the circular patches visualized by dye (see section 4.3 for details of image processing). To compare $R$ with $L$, we performed a complementary experiment where both techniques are applied together: visualization of the injected water and PIV measurement. Figure 7 shows that the two values agree by $10 \%, R$ being larger than $L$. Thus a dividing factor of 1.1 has been applied in the following to all our radius $R$ measurements of the dye patches in order to transform these radii $R$ into the proper typical horizontal scales $L$. In particular, to build the state diagram of figure 16, the initial values of $R$ will be transformed in initial values of $L$ in order to compare our experimental data to former published results.

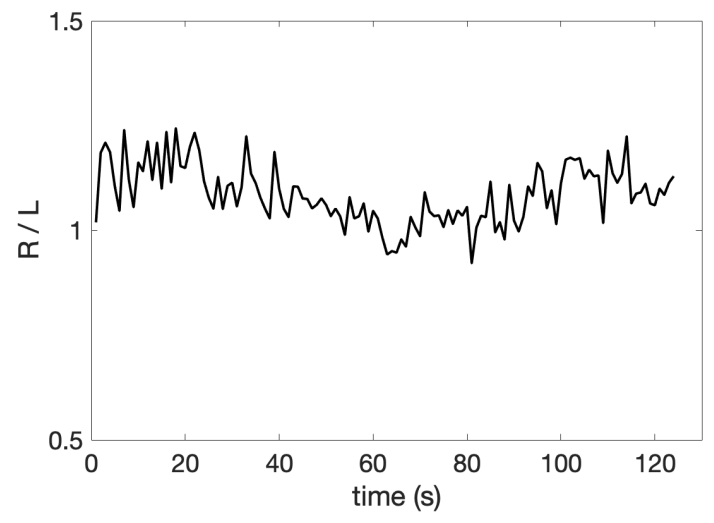

Figure 7. Temporal evolution of the ratio $R / L$ for a single vortex. 
The height $H$ of the vortices are directly measured by image analysis of the videos recorded by the side-view camera to calculate the Rossby deformation radius $\lambda$. We will keep the initial values of these heights i.e. after the transients due to the time needed for the anticyclones to reach their geostrophic balance (neglecting the presence of the other vortex in the pair) to perform this calculation.

\section{Coalescence of identical anticyclones}

\subsection{Visualization of the merging process}

As explained previously, the vortices are created by injection of 5 to $20 \mathrm{ml}$ of salt water in a layer that was stratified in density with salt and set into rotation for two hours before performing each experiment. The density of the injected water was chosen to be the value of the density at the injection location so that the anticyclones stay at the desired altitude. The top-view camera records the complete process from the injection to the final time when all the fluid motions are damped out, while the side-view camera records only the first minutes of the runs. A complete sequence lasts from 3 to 30 minutes depending upon the initial conditions. Figure 8 presents for $f=1.57 \mathrm{rad} / \mathrm{s}, N=2.8 \mathrm{rad} / \mathrm{s}, d_{0}=8 \mathrm{~cm}$ and $Q=45 \mathrm{ml} / \mathrm{min}$, four images from a typical sequence where two anticyclones merge. The first effect of the mutual influence of one vortex onto the other is a global rotation of the pair. Then, when getting closer to each other, the vortices are deformed and a cusp is formed on the inner sides of both of them. The streaklines around these cusps are then elongated, captured and then wrapped around the opposite core to form a Yin and Yang Chinese symbol. As observed on the pictures, the process is threedimensional as the vortices intertwine with overlapping of both cores seen as a superimposition of red and green colors. We also note the presence of dye filaments repulsed away from the final merged vortex core.
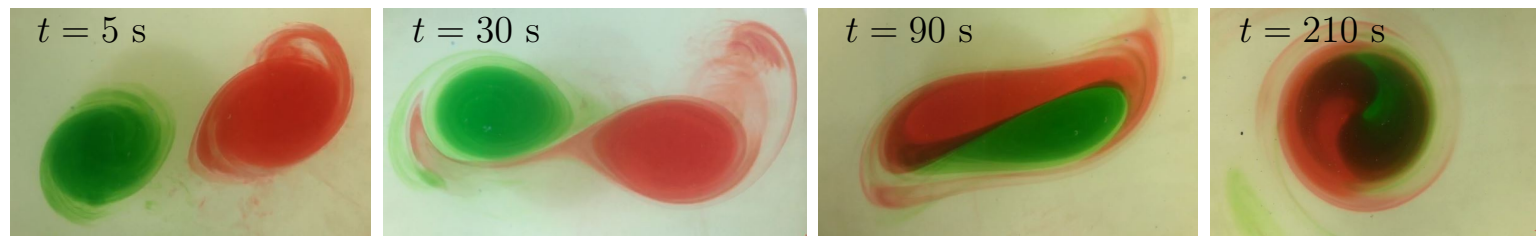

Figure 8. Temporal sequence showing the coalescence of two anticyclones for $f=1.57 \mathrm{rad} / \mathrm{s}, N=2.8 \mathrm{rad} / \mathrm{s}, Q=45$ $\mathrm{ml} / \mathrm{min}$ and $d_{0}=8 \mathrm{~cm}$.

Figure 9 presents another illustration of the merging process in the case where the cyclonic shields are repulsed in the form of two side cyclonic vortices. For this sequence, $f=1 \mathrm{rad} / \mathrm{s}$, $N=3.12 \mathrm{rad} / \mathrm{s}, d_{0}=7.5 \mathrm{~cm}$ and $Q=25 \mathrm{ml} / \mathrm{min}$. These visualizations are perfect illustrations of the numerical simulations of Valcke and Verron (1997) for two layers quasi-geostrophic models and of Ciani et al. (2016) for computations of realistic oceanographic eddy coalescence.

The three-dimensional features of the merging of baroclinic vortices can also be observed by the side-view camera. Figure 10 presents two images where the pair of anticyclones is viewed from the side for two different instants. It is very clear on these images that the vortices are tilted (versus the vertical axis) during coalescence. Although it is difficult to prove, we speculate that an elliptic instability may take place here in the same way it appears in the case of barotropic columnar vortices merging (Meunier et al. 2005). Unlike the elliptic instability of these columnar vortices, the most unstable mode of the elliptic instability of fluids inside rotating ellipsoids (Lacaze et al. 2004) is a rotation around a tilted axis (the spin-over mode). From the top-view images, it is clear that the mutual interaction between the anticyclonic lenses 

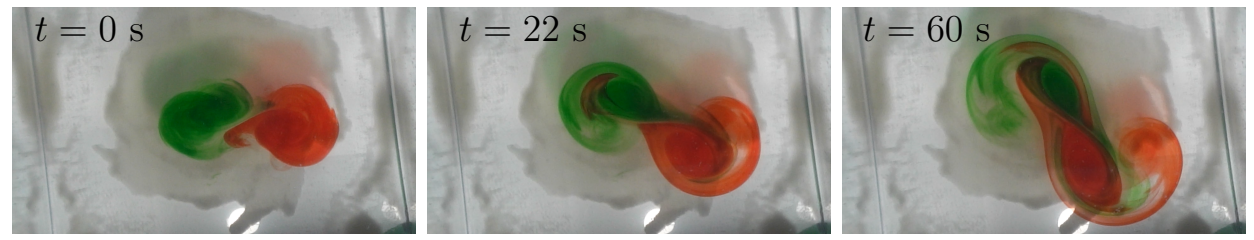

Figure 9. Temporal sequence showing the coalescence of two anticyclones for $f=1 \mathrm{rad} / \mathrm{s}, N=3.12 \mathrm{rad} / \mathrm{s}, d_{0}=7.5 \mathrm{~cm}$ and $Q=25 \mathrm{ml} / \mathrm{min}$. Two side cyclones coming from the cyclonic rings are ejected from the merging anticyclonic pairs.

can deform sufficiently each vortex such that their ellipticity is large and of the order of their diameter, enhancing the possible appearance of the instability in this free of boundary layers flow.
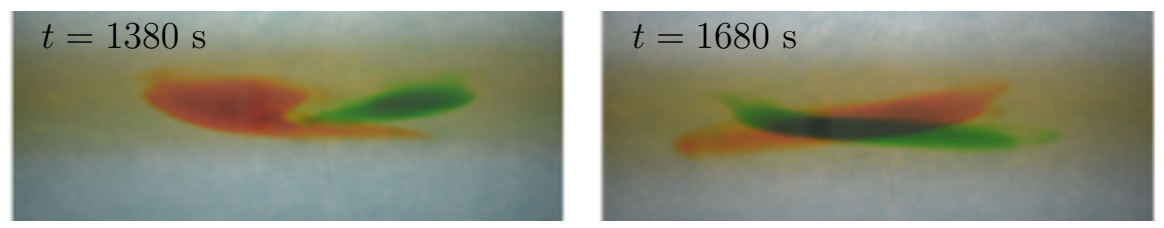

Figure 10. Two observations of the merging process from the side view camera for $f=1 \mathrm{rad} / \mathrm{s}, N=3.16 \mathrm{rad} / \mathrm{s}, Q=30$ $\mathrm{ml} / \mathrm{min}$ and $d_{0}=14 \mathrm{~cm}$. The anticyclonic lenses are clearly tilted when merging, enhancing the three-dimensional process as they intertwine each other. We suspect the elliptic instability to be at the origin of this tilting.

\subsection{PIV measurements of coalescence}

Besides visualization, we have also performed PIV measurements of velocity fields during coalescence. Figure 11 presents an example of the temporal evolution of a vorticity field. At initial times, soon after the vortex generation, we can see the separated cores and their shielding cyclonic rings which, in the present case, have merged in a figure eight shape. Rapidly this shield disappears and as the vortices rotate one around the other, two vorticity arms are formed at the external tips of the pair. These arms wrap around the vortices and become thinner as they are elongated by the core rotation. Finally, these filaments are dissipated while the cores are completely merged.

\subsection{Image analysis and measurements of the global induced rotation rate and the merging time of the pair}

As mentioned before we have tracked the vortices visualized with dye by image analysis. We first determine the center position of each vortex and measure their radius by extracting one frame per second from the video. A red and then a green (or blue, according to the dye color we used) filter is applied to each frame. An intensity filter for each color is then used in order to highlight each vortex. These highlighted images are then binarized such that only the vortices are visible. From these images we can now extract different parameters like the radius and the center position of each vortex. These radii are calculated such that the corresponding disks have the same areas as the binarized patches. The vortex common rotation center is then determined and we can compute the global orbital rotation rate $\omega_{\text {orb }}$ of the pair and the separation distance between both vortices. Figure 12 shows an example of the merging process for $f=0.5 \mathrm{rad} / \mathrm{s}$, $N=1.75 \mathrm{rad} / \mathrm{s}, Q=60 \mathrm{ml} / \mathrm{min}$ and $d_{0}=8 \mathrm{~cm}$ on which we have applied our image analysis. As can be observed, in this case, both vortices have ejected their cyclonic rings in the form 

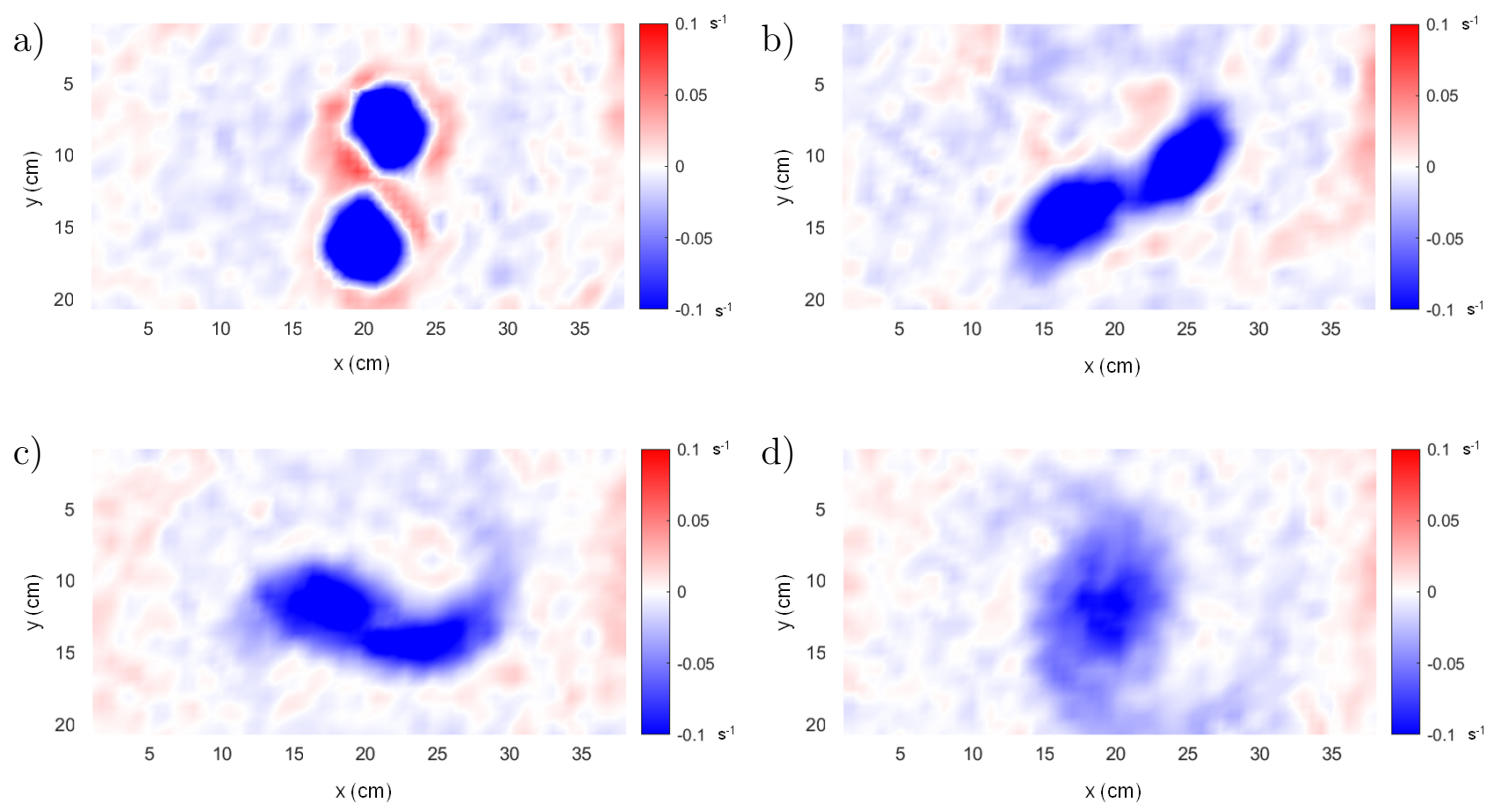

Figure 11. Temporal sequence showing the vorticity fields during the coalescence of two anticyclones for $f=0.5 \mathrm{rad} / \mathrm{s}$, $N=0.95 \mathrm{rad} / \mathrm{s}, Q=60 \mathrm{ml} / \mathrm{min}$ and $d_{0}=8 \mathrm{~cm}$ for: a) $t=1 \mathrm{~s}$, b) $t=30 \mathrm{~s}$, c) $t=90 \mathrm{~s}$ and d) $t=200 \mathrm{~s}$. Filaments are formed at the external tips of the vortices and will eventually be dissipated whereas the cores are merged in a unique vortex.

of two thin cyclonic filaments. We have also represented on the figure the white circles whose centers and radii have been determined by our image processing.
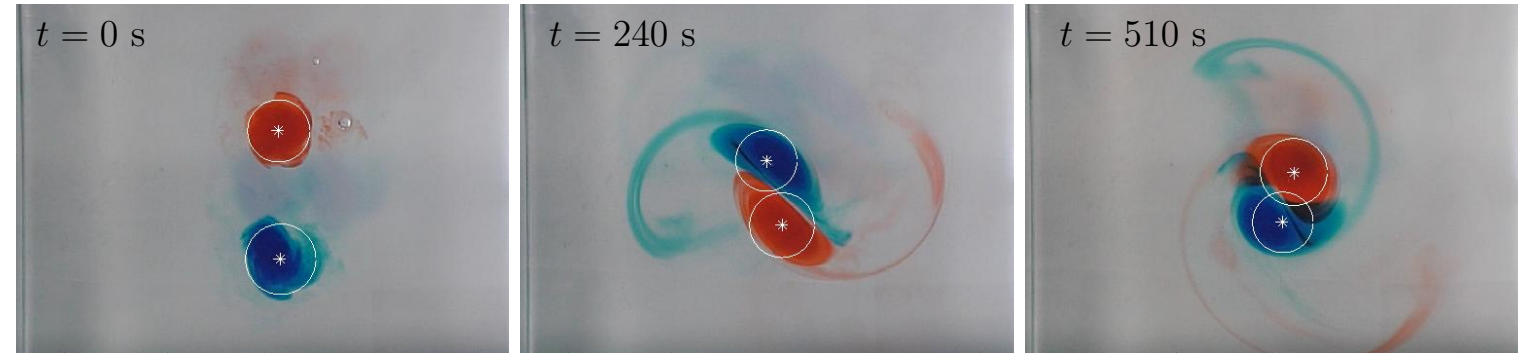

Figure 12. Temporal sequence showing the coalescence of two anticyclones for $f=0.5 \mathrm{rad} / \mathrm{s}, N=1.75 \mathrm{rad} / \mathrm{s}, Q=60$ $\mathrm{ml} / \mathrm{min}$ and $d_{0}=8 \mathrm{~cm}$. The white circles and stars indicate the vortex centers and their corresponding radii found via image processing.

Figure 13-a) shows the spiraling trajectories of each vortex in the rotating frame of the table; the numbers beside the plotted points indicate the elapsed time in seconds. From these, we can easily calculate the azimuthal positions $\theta_{1}$ and $\theta_{2}$ of each vortex core versus time, and deduce by a simple linear fit the orbital rotation rates $\omega_{1}$ and $\omega_{2}$ of each vortex as shown in figure 13-b). As $\omega_{1}$ and $\omega_{2}$ have nearly constant and very close values in all the runs, we will, in the following, average them to obtain a measurement of the global orbital rotation rate $\omega_{\text {orb }}$. We note a systematic slight increase of $\omega_{\text {orb }}$ at the end of the merging processes. In the same way, we can measure the distance between the cores as a function of time. Figure 13-c) presents these results from the former example.

As can be observed, we can systematically identify three different stages in the merging process in accordance to what has been previously described in the literature for barotropic vortex coalescence (Melander et al. 1987, Meunier et al. 2005, Leweke et al. 2016). At the beginning 

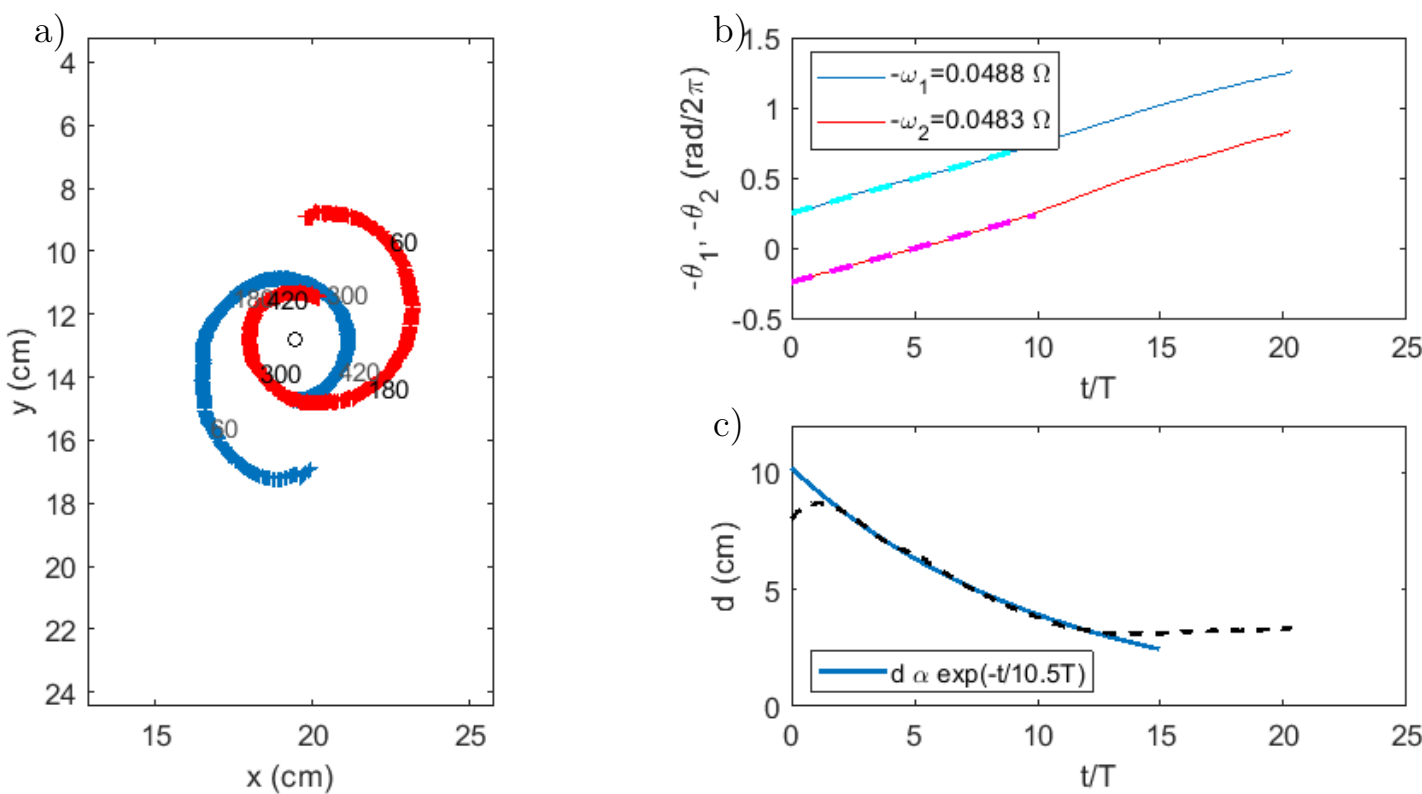

Figure 13. a) The spiral trajectories of the vortices as function of time for the merger of figure 12. b) Temporal evolution of the azimuthal angle of both vortices for the merger of figure 12; time is given in rotating table period and $-\omega_{1}$ and $-\omega_{2}$ are the global rotation rates of each vortex measured by the linear fit at the initial time (dashed lines). c) Temporal evolution of the distance $d$ between both vortices for the merger of figure 12; We have also represented the exponential fit of this evolution that gives an estimation of the merging time $\tau$ (in this case $\tau=10.5 T$ ).

of the interaction we see that the distance between centers stays constant or varies slightly; this first period of time could be identified as a first diffusive stage. In the second or convective stage a rapid decrease of the separation distance is observed until it reaches a minimum. In the third stage, the vortices have merged and we no longer detect any variation of the distance between their centers. To characterize the merging time, we choose to fit the intermediate (or convective stage,where each vortex feels the velocity field from the other vortex that induces the merging) by a decreasing exponential function of time $\exp (-t / \tau)$ where $\tau$ represents the characteristic time of coalescence. This method permits to deduce easily and automatically $\tau$ in order to describe the duration of the process as function of the experimental parameters.

We have found that both the global rotation rate $\omega_{\text {orb }}$ of the vortex pair and the merging time $\tau$ are influenced by the injection flux $Q$ that creates the vortices. A crude two-dimensional model can be built by applying Stoke's theorem on a circle passing through the centers of both vortices: it is straightforward to show that the global orbital rotation rate $\omega_{o r b}$ is proportional to the vorticity contained essentially in the vortex cores of radius $R$ and inversely proportional to the square of the initial separating distance $d_{0}$. As the Rossby number of each vortex is itself a linear function of the injection rate $Q$ (see figure 5), we can plot $\omega_{\text {orb }}$ as a function of $Q R^{2} / d_{0}^{2}$ to test this prediction. Figure 14 shows that this trend is verified by our experimental data: the larger the radii and the smaller the initial distances are, the higher the orbital angular velocity of the vortices around their barycenter is. We can also observe on figure 14 that high injection fluxes $Q$ correspond to fast angular velocities. This can be understood using the already mentioned link between the Rossby number of each vortex and the injection rate through the Coriolis force action. We can thus conclude that the individual angular velocity measured by the Rossby number of each vortex has a direct effect on the global rotation rate of the pair of vortices.

In the same spirit, we have also found that the injection flux $Q$ directly influences the merging time $\tau$. This is shown in figure 15. As expected, the larger the initial distance and the smaller the radii are, the longer it takes for the vortices to merge. Interestingly, we can also observe 


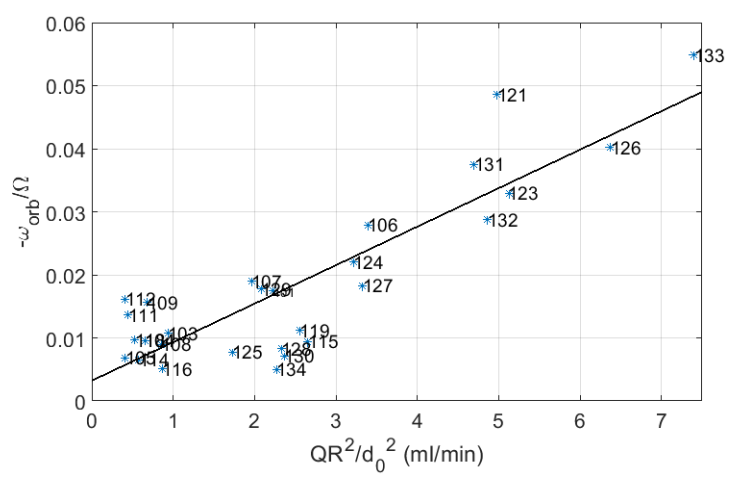

Figure 14. The evolution of the orbital rotation rate $-\omega_{\text {orb }}$ as function of $Q R^{2} / d_{0}{ }^{2}$ where $R$ is the radius of the vortex cores, $d_{0}$ the initial separating distance, and $Q$ the injection flux which is proportional to the Rossby number. The linear trend as expected by a crude two-dimensional model is recovered.

that mergers are faster $(\tau$ small $)$ for high injection flux $Q$, i. e. is for large individual Rossby numbers. The role of the Rossby number in the coalescence process has also been discovered by the recent numerical observations of Reinaud and Dritschel (2018).

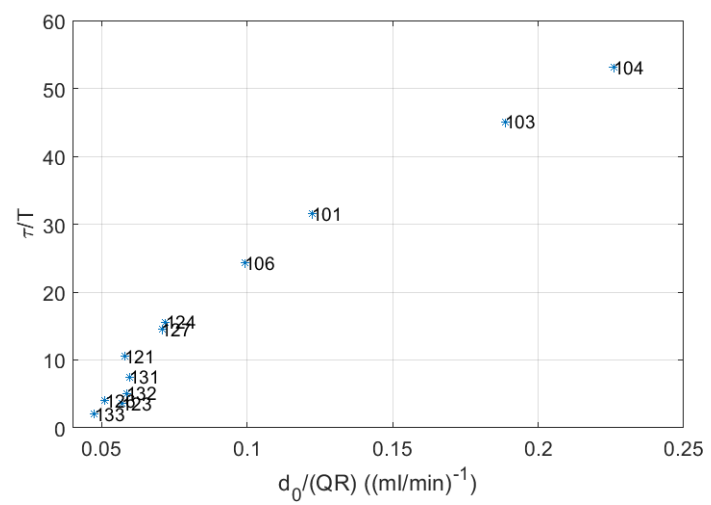

Figure 15. The evolution of the merging time as function of $Q d_{0} / R$ where $R$ is the radius of the vortex cores, $d_{0}$ the initial separating distance, and $Q$ the injection flux which is proportional to the Rossby number. This shows in particular that the merging time depends on the vortex individual Rossby number.

\subsection{The state diagram}

Having varied the initial distance $d_{0}$ separating the vortices at their creation, the rotating rate $\Omega$ of the rotating table, the density gradient that implies different Brünt-Väisälä frequencies and also the injection rate $Q$ that permits to change the Rossby number and thus the initial dimensions $H$ and $L$ of each vortical pairs, we can build the state diagram in the $(\lambda / L$ and $\left.d_{0} / L\right)$ plane. When pairing is observed, we represent the result at its corresponding location with a blue star next to its label. When the vortices do not merge, we plot the result as a red circle. Each experiment is labeled with a number which is smaller than 100 for hand injection runs and above 100 for pump injection runs. Figure 16 presents this synthesis. As expected, the blue stars are concentrated at the bottom of the figure, i.e. when the initial separating distance $d_{0}$ is small. We have shaded this area in blue. On the contrary, when the initial distance is large, the vortices have more difficulty to merge and the red stars are mostly located in the upper part of the figure. However, it is also clear from our results that the Rossby radius of deformation has a tremendous influence on coalescence. Although we cannot perform experiments at too 
small values of $\lambda / L$ (our lowest $\lambda / L$ is around 0.6) because of the presence of centrifugal or baroclinic instabilities that destroy individual vortices, we observe that our experimental results are compatible with the two-dimensional limit. Together with our experimental data points, we represent also the different results from diverse authors. First, the Griffiths and Hopfinger's curve (1987) is plotted (dashed blue curve). It presents a clear increase of the critical distance when $\lambda / L$ is between 2 and 4 . As can be observed on figure 16, our experimental data points show a similar trend when $\lambda / L$ is between 1 and 3 . Around a value close to 2.5 , the vortices are prone to merge even if they are separated by a distance more than twice the two-dimensional critical distance $(7.5 \times L$ compared to $3.3 \times L)$. However, contrary to Griffiths and Hopfinger's results but in excellent agreement with the numerical simulations of Verron et al. (1990) (dashdotted red curve), our experimental critical curve obviously decreases back when $\lambda / L$ is larger than 3. Then for $\lambda / L$ larger than 4, i.e. with strong stratification effects compared to rotation, the vortices and their motions are confined in very shallow layers and the frontier $d_{c}$ between pairing and non pairing dynamics decreases back to its expected two-dimensional value close to 3.3. Note that our results do not support (as expected) the results from the three-dimensional quasi-geostrophic model of Dritschel (2002) (solid black curve) where a secondary instability on tall vortices, not seen in our experiments, was suspected to help coalescence. Amazingly, our results appear to match better with the RVI (see the definition of RVI and PVI in our introduction) two-layer model of Verron et al. (1990) than with the two-layer Griffiths and Hopfinger's experiments. Verron et al. discuss that the initial conditions of the Griffiths and Hopfinger's experiments are more consistent with PVI conditions because the injection of fluid in the upper layer would deflect the interface and according to potential vorticity conservation, some relative vorticity should be created in the lower layer. With this argument, they reach some kind of contradiction in the sense that the results from the Griffiths and Hopfinger's experiments are more consistent with RVI type conditions contrarily to their initial conditions which are more consistent with PVI type conditions. They try to explain this contradiction by stating that the vorticity induced in the lower layer is weaker than in the upper layer thus the vortices do not present a barotropic vertical structure. They think that the vorticity field in the lower layer is weak enough to prevent the initial conditions to be entirely of PVI type and that would be the reason why the Griffiths and Hopfinger's experimental results differ from the PVI results. In our case, the linear stratification might act as a shield, isolating the vortices from the fluid above and under the vortices. Therefore the relative vorticity stays zero outside the vortices as it can be observed on figure 6 . As a consequence, our initial conditions are very close to RVI conditions, and this explains why our experimental results are so similar to the RVI numerical simulations of Verron et al. (1990).

To our point of view, the final picture of baroclinic vortex coalescence could be interpreted as follows. For $\lambda / L$ lower than unity (in this case the Rossby radius of deformation stands inside the vortex core), the influence of density stratification is weak and the critical distance $d_{c}$ stays close to the two-dimensional limit $3.3 \times L$. Note that in the experiment by Griffiths and Hopfinger (1987) as well as in the numerical simulations of Verron et al. (1990) and also of Dritschel (2002), there is a small decrease of this limit down to $2.5 \times L$. Then, for $\lambda>L$, as we saw previously, stratification helps coalescence. If we consider that the radius of influence of a vortex is equal to the Rossby radius of deformation (a scale lager than the vortex core radius $L$ in this case) we propose to replace the barotropic criterion $d_{c}=3.3 \times L$ by $d_{c}=3.3 \times \lambda$ which gives a rather good estimation of the frontier separating pairing to non pairing vortices. Finally, as already noted, coalescence being a three dimensional process, a further increase of the density gradients (and thus of $N$ ) makes vortex pairing more and more difficult. The limit is reached in our experiment for $\lambda / L \simeq 3$ where the critical distance starts to decrease back towards its two-dimensional value, reached as expected for very flat pairs of anticyclones in highly stratified fluid layers. This return to the barotropic value at large $\lambda / L$ was not observed in the experiment of Griffiths and Hopfinger (1987) possibly because of the two layer configuration that confines 


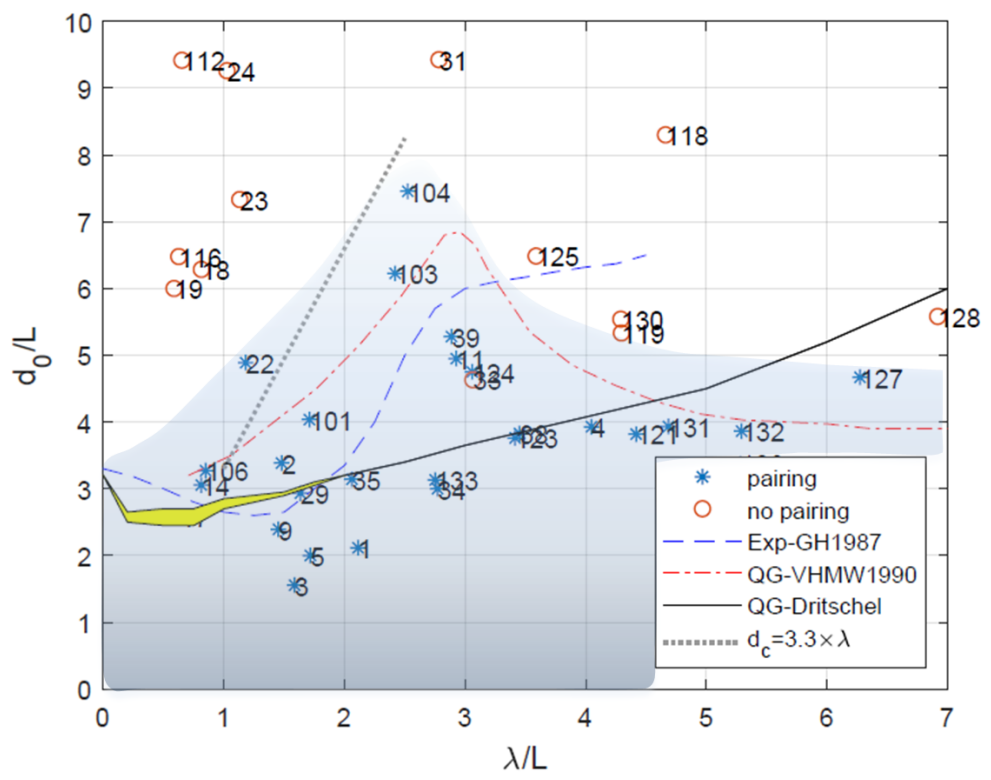

Figure 16. State diagram showing a synthesis of our experiments with the pairing $(\star)$ and the non pairing $(\circ)$ events together with the different numerical predictions of Verron et al. (1990) (- - -) and of Dritschel (2002) (-). Note that in accordance with the definition of the Rossby radius of deformation used by Verron et al. (1990) and explained in Hopfinger and van Heijst (1993), the red dash-dotted curve has been shifted by a multiplicative factor $\sqrt{2}$. The yellow area represents the "partial pairing" region detected by Dritschel (2002). The experimental two layer experiment results of Griffiths and Hopfinger (1987) are represented by the blue dash curve (- - ) and our estimation of the extension of the barotropic criterion by the grey dotted curve $(\cdots)$.

the vortices in the top layer.

\section{Fourier modes of the merger and Internal Gravity Waves radiation}

Internal Gravity Waves (IGWs) play a major role in the atmosphere and in the ocean where they can be seen as a conveyer of energy from large scale flows to small scales so that they participate to mixing and energy dissipation. Therefore, many studies focus on the spontaneous generation of IGWs from nearly balanced flows (see the review by Vanneste (2013)). Among these, several numerical simulations based on primitive equation simulations (Sugimoto and Plougonven 2016) or based on shallow water models (Sugimoto et al. 2015, Sugimoto 2015) show that vortex dipoles or vortex pairs are prompt to generate IGWs. The ultimate goal of these researches is to quantify the amount of ageostrophic energy loss when the vortices are propagating in the case of a dipole, or merging in the case where they are of the same sign. Therefore, in addition to describing the different regimes that lead or not to coalescence, we will address now the problem of the generation of IGWs during the transient time of the fusion of our lenticular anticyclonic pairs. These were already observed in the numerical simulations of Viudez (2006), Sugimoto (2015) and of Reinaud and Dritschel (2018). A classical procedure to detect Internal Gravity Waves (IGWs) is to calculate the horizontal divergence of the PIV velocity fields (Sugimoto 2015, Rodda et al. 2018). Then a space-time diagram can be computed by recording the fluctuations of the horizontal divergence along a line going through the pair of vortices (see figure 17-a for an example with $f=0.5 \mathrm{rad} / \mathrm{s}$ and $N=0.95 \mathrm{rad} / \mathrm{s}$ ). As can be observed on figure 17-b), waves clearly propagate away from the merging pair, as expected for $f<N$. The calculation of the average period of these waves (about 10 seconds) show that their frequency is equal to $0.62 \mathrm{rad} / \mathrm{s}$, a value between $f$ and $N$ as requested by the dispersion relation of IGWs. Note that their wavelength is comparable with the diameter of the initial vortices. As the sign of the phase velocity is a function of $f-N$, we also present in figure 17-c) 
an example of inwards propagation for $f=1.2 \mathrm{rad} / \mathrm{s}, N=0.95 \mathrm{rad} / \mathrm{s}, Q=60 \mathrm{ml} / \mathrm{min}$ and $d_{0}=8 \mathrm{~cm}$, i.e. in the case where $f>N$.
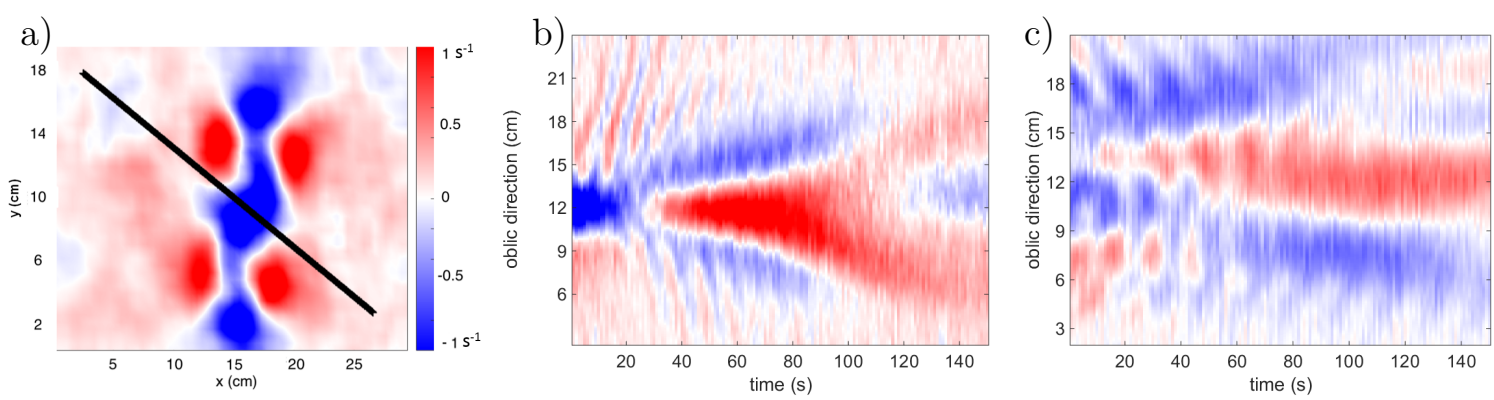

Figure 17. a) Horizontal divergence of a PIV velocity field for $f=0.5 \mathrm{rad} / \mathrm{s}, N=0.95 \mathrm{rad} / \mathrm{s}, Q=60 \mathrm{ml} / \mathrm{min}$ and $d_{0}=8 \mathrm{~cm}$, with the intersecting line along which the space-time diagram is computed. b) Space-time diagram computed along the preceding line passing through the vortex pair. Wave trains are clearly observed with a period of 10 seconds, compatible with the outwards propagation of IGWs. c) Space-time diagram showing inwards propagation of waves for $f=1.2 \mathrm{rad} / \mathrm{s}, N=0.95 \mathrm{rad} / \mathrm{s}, Q=60 \mathrm{ml} / \mathrm{min}$ and $d_{0}=8 \mathrm{~cm}$.

Figure 18 shows the temporal Fourier spectrum of a time series recorded at a point in one of our PIV horizontal divergence fields for $f=1 \mathrm{rad} / \mathrm{s}, N=0.7 \mathrm{rad} / \mathrm{s}, Q=25 \mathrm{ml} / \mathrm{min}$ and $d_{0}=5 \mathrm{~cm}$. The low frequency peaks correspond to the different Fourier modes with different azimuthal wave numbers $m$ associated with the double structure of the field during the vortex pairing. In addition a well defined wave packet is clearly visible in the IGWs authorized band between the buoyancy frequency $N$ and the Coriolis frequency $f$. Snapshots of these modes and of the propagating IGWs fields are calculated by band-pass filtering the global vorticity field. The low frequency modes are associated with the deformation of the vorticity field due to the mutual induction of the vortices in the pair. All these modes rotate with an identical rotation rate which is that of the vortices orbiting one around the other at the rate $\omega_{\text {orb }}$ while the IGWs propagate radially with their own phase velocity. Let us note however that if our observations of IGWs wave packets are in agreement with recent numerical computations of Viudez (2006) and of Reinaud and Dritschel (2018), we are not able at the stage of our study to separate the IGWs wave trains created by the injection process when generating the vortices to those which can be naturally emitted during merging by the rotating non axi-symmetric vortical patterns.

\section{Conclusion}

In this experimental work, we have for the first time studied the coalescence of baroclinic anticyclones that were generated in a rotating and continuously stratified layer. Our results clearly show that the merging process is highly dependent on the Rossby radius of deformation $\lambda$. First, when $\lambda$ is smaller than the vortex core, stratification seems not to play an important role. Then, in a range of $\lambda$ between 1 and 3, coalescence is greatly enhanced. Our interpretation of this enhancement suggests a modification of the two-dimensional criterion by the replacement of the anticyclones radius by their Rossby radius of deformation as this horizontal length is the effective scale associated to baroclinic vortices. But this positive effect due to stratification is only efficient in a restricted region of $\lambda / L$. As coalescence of baroclinic vortices involves three dimensional flows during which the vortices intertwine possibly because of the elliptic instability, it is expected that very flat vortices in highly stratified fluid resist against merging and the twodimensional limit is recovered. As a consequence, the barotropic criterion is recovered at large $\lambda$. We have also shown that the merging time and the global rotation rate of the pair depend on the Rossby number of each vortex. Besides the description of the state diagram that confirms 


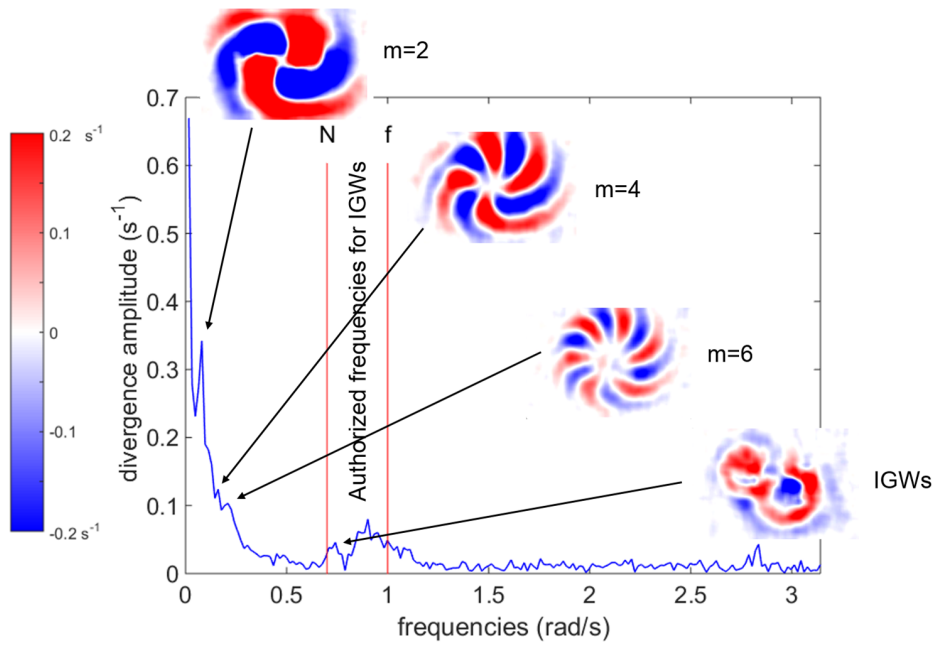

Figure 18. Fourier spectrum of a temporal series recorded at a point in the PIV horizontal divergence field together with the spatial structures of the corresponding modes labeled by their azimuthal wave numbers $m$ involved in the vortex merging. The internal gravity waves (IGWs) packet emitted during the merging is visible in the authorized spectral band confined between $N$ and $f$.

the numerical simulation of Verron et al. (1990), we have also discovered that internal gravity waves are emitted during mergers. Unfortunately, we cannot discriminate at this point the waves generated by the injections used to create the vortices, to those that are emitted by the non balanced vortex pair during coalescence. As a final point, let us mention the visual analogy of our hydrodynamical system with the merger of two black holes associated with the radiation of gravitational waves (Abbott et al. 2016).

\section{Acknowledgments}

This work has been carried out in the framework of the Labex MEC Project 410 (No. ANR10-LABX-0092) and of the A*MIDEX Project (No. ANR-11-IDEX- 0001-02), funded by the "Investissements d'Avenir" French Government program managed by the French National Research Agency (ANR). The authors also acknowledge support from the American Physical Society's International Research Travel Award Program (IRTAP). We also thank for their financial support, the University of Guadalajara and Aix Marseille Université for the fundings "Appel à Projets Actions Internationales" 2016. A. O. E. acknowledges CONACyT for his scholarship.

\section{References}

Abbott, B.P., and LIGO Scientific Collaboration and Virgo Collaboration. Observation of gravitational waves from a binary black hole merger. Phys. Rev. Lett., 2016, 116, 061102.

Aubert, O., Le Bars, M., Le Gal, P. and Marcus, P., The universal aspect ratio of vortices in rotating stratified flows: experiments and observations. J. Fluid Mech., 2012, 706, 34-45.

Barbosa, A., Ana, C., Peliz, Á. and Carton, X., A census of meddies in a long-term high-resolution simulation. Prog. Oceanogr., 2013, 116, 80-94.

Billant, P., Zigzag instability of vortex pairs in stratified and rotating fluids. Part 1. General stability equations. J. Fluid Mech., 2010, 660, 354-395.

Cariteau, B., Etude de la stabilité et de l'interaction de cyclones intenses en fluide stratifié. Ph.D. Thesis, Université Joseph Fourier (Grenoble), 2005.

Carton, X., Chérubin, L., Paillet, J., Morel, Y., Serpette, A. and Le Cann, B., Meddy coupling with a deep cyclone in the Gulf of Cadiz. J. Mar. Syst., 2002, 32, 13-42.

Carton, X., Daniault, N., Alves, J., Cherubin, L. and Ambar, I., Meddy dynamics and interaction with neighboring eddies southwest of Portugal: observations and modeling. J. Geophys. Res. Ocean, 2010, 115. 
Charney, J.G., Geostrophic Turbulence. J. Atmos. Sci., 1971, 28, 1087-1095.

Ciani, D., Carton, X. and Verron, J., On the merger of subsurface isolated vortices. Geophys. Astrophys. Fluid Dyn., 2016, 110, 23-49.

Cushman-Roisin, B., On the role of filamentation in the merging of anticyclonic lenses. J. Phys. Oceanogr., 1989, 19, 253-258.

De la Rosa Zambrano, H.M., Cros, A., Cruz Gómez, R.C., Le Bars, M. and Le Gal, P., A laboratory study of floating lenticular anticyclones. Eur. J. Mech. - B/Fluids, 2017, 61, 1-8.

Dritschel, D.G., Vortex merger in rotating stratified flows. J. Fluid Mech., 2002, 455, 83-101.

Facchini, G. and Le Bars, M., On the lifetime of a pancake anticyclone in a rotating stratified flow. J. Fluid Mech., 2016, 804, 688-711.

Griffiths, R.W. and Hopfinger, E.J., Coalescing of geostrophic vortices. J. Fluid Mech., 1987, 178, 73-97.

Hassanzadeh, P., Marcus, P.S. and Le Gal, P., The universal aspect ratio of vortices in rotating stratified flows: theory and simulation. J. Fluid Mech., 2012, 706, 46-57.

Hopfinger, E.J. and van Heijst, G.J.F., Vortices in rotating fluids. Ann. Rev. Fluid Mech., 1993, 25, 241-289.

Kraichnan, R.H., Inertial ranges in two dimensional turbulence. Phys. Fluids, 1967, 10, 1417-1423.

Lacaze, L., Le Gal, P. and Le Dizès, S., Elliptical instability in a rotating spheroid. J. Fluid Mech., 2004, 505, $1-22$.

Leweke, T., Le Dizès, S. and Williamson, C.H.K., Dynamics and instabilities of vortex pairs. Ann. Rev. Fluid Mech., 2016, 48, 507-541.

L'Hégaret, P., Carton, X., Ambar, I., Ménesguen, C., Hua, B.L., Chérubin, L., Aguiar, A., Cann, B., Daniault, N. and Serra, N., Evidence of Mediterranean water dipole collision in the Gulf of Cadiz. J. Geophys. Res. Ocean, 2014, 119, 5337-5359.

Melander, M.V., McWilliams, J.C. and Zabusky, N.J., Axisymmetrization and vorticity-gradient intensification of an isolated two-dimensional vortex through filamentation. J. Fluid Mech., 1987, 178, 137-159.

Melander, M.V., Zabusky, N.J. and McWilliams, J.C., Symmetric vortex merger in two dimensions: causes and conditions. J. Fluid Mech., 1988, 195, 303-340.

Meunier, P., Le Dizès, S. and Leweke, T., Physics of vortex merging. C. R. Phys., 2005, 6, 431-450.

Nielsen, A.H., He, X., Rasmussen, J.J. and Bohr, T., Vortex merging and spectral cascade in two-dimensional flows. Phys. Fluids, 1996, 8, 2263-2265.

Oster, G., Density gradients. Sci. Am., 1965, 213, 70-79.

Otheguy, P., Chomaz, J.M., Augier, P., Kimura, Y. and Billant, P., Pairing of two vertical columnar vortices in a stratified fluid. Eur. J. Mech. - B/Fluids, 2015, 49, 413-425.

Polvani, L.M., Zabusky, N.J. and Flierl, G.R., Two-layer geostrophic vortex dynamics. Part 1. Upper-layer V-states and merger. J. Fluid Mech., 1989, 205, 215-242.

Reinaud, J.N. and Dritschel, D., The merger of geophysical vortices at finite Rossby and Froude number. J. Fluid Mech., 2018, 848, 388-410.

Rodda, C., Borcia, I.D., Le Gal, P., Vincze, M. and U., H., Baroclinic, Kelvin and inertia-gravity waves in the barostrat instability experiment. Geophys. Astrophys. Fluid Dyn., 2018, 112, 175-206.

Rodríguez-Marroyo, R., Viúdez, Á. and Ruiz, S., Vortex merger in oceanic tripoles. J. Phys. Oceano., 2011, 41, 1239-1251.

Sanchez-Lavega, A., Orton, G.S., Morales, R., Lecacheux, J., Colas, F., Fisher, B., Fukumura-Sawada, P., Golisch, W., Griep, D., Kaminski, C. et al., The merger of two giant anticyclones in the atmosphere of Jupiter. Icarus, 2001, 149, 491-495.

Schultz Tokos, K.L., Hinrichsen, H.H. and Zenk, W., Merging and migration of two meddies. J. Phys. Oceano., 1994, 24, 2129-2141.

Sugimoto, N., Inertia-gravity wave radiation from the merging of two co-rotating vortices in the f-plane shallow water system. Phys. Fluids, 2015, 27, 121701.

Sugimoto, N., Ishioka, K., Kobayashi, H. and Shimomura, Y., Cyclone-anticyclone asymmetry in gravity wave radiation from a co-rotating vortex pair in rotating shallow water. J. Fluid Mech., 2015, 772, 80-106.

Sugimoto, N. and Plougonven, R., Generation and backreaction of spontaneously emitted inertia-gravity waves. Geophys. Res. Lett., 2016, 43, 3519-3525.

Thielicke, W. and Stamhuis, E., PIVlab-towards user-friendly, affordable and accurate digital particle image velocimetry in MATLAB. J. Open Res. Soft., 2014, 2.

Valcke, S. and Verron, J., Interactions of baroclinic isolated vortices: the dominant effect of shielding. J. Phys. Oceano., 1997, 27, 524-541.

Vanneste, J., Balance and spontaneous wave generation in geophysical flows. Ann. Rev. Fluid Mech., 2013, 45, $147-172$.

Verron, J., Hopfinger, E.J. and McWilliams, J.C., Sensitivity to initial conditions in the merging of two-layer baroclinic vortices. Phys. Fluids A: Fluid Dyn., 1990, 2, 886-889.

Verron, J. and Valcke, S., Scale-dependent merging of baroclinic vortices. J. Fluid Mech., 1994, 264, 81-106.

Viudez, A., Spiral patterns of inertia-gravity waves in geophysical flows. J. Fluid Mech., 2006, 562, 73-82.

Von Hardenberg, J., McWilliams, J.C., Provenzale, A., Shchepetkin, A. and Weiss, J.B., Vortex merging in quasi-geostrophic flows. J. Fluid Mech., 2000, 412, 331-353.

Waugh, D.W., The efficiency of symmetric vortex merger. Phys. Fluids A: Fluid Dyn., 1992, 4, 1745-1758.

Zabusky, N.J., Hughes, M.H. and Roberts, K.V., Contour dynamics for the Euler equations in two dimensions. J. Comp. Phys., 1979, 30, 96-106. 